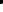


Grumman Research Department Memorandum RM-323J

\section{POLARIMETRIC MEASUREMENTS OF SIMULATED LUNAR SURFACES ${ }^{\dagger}$}

by

W. G. Egan

and

L. L. Smith

Geo-Astrophysics Section

April 1966

tPresented at the $121^{\text {st }}$ Meeting of the American Astronomical Society, March 28-31, 1966, NASA Langley Research Center, Hampton, Virginia. 


\section{ABSTRACT}

Polarimetric studies of rough surfaces serve to delimit the range of their appropriate physical properties and geometries. In an effort to lay the basis for an analytical approach, the polarization properties of volcanic ash and furnace slag were investigated as a function of particle size, albedo, and porosity; current theories of polarization indicate that these properties are significant. These investigations were made on a large scale polarimeter (4-inch diameter viewing area) so that coarse specimens could be analyzed.

For both samples, it was found that the maximum percent polarization (positive) and the corresponding phase angle at zero and $60^{\circ}$ viewing angles, the inversion angle and the minimum percent polarization (negative) varied as a function of porosity and a1bedo. It was found that the best fit to Crisium (Gehrels et a1.) could be achieved with Haleakala volcanic ash with particle sizes between 37 and 88 microns, and furnace slag less than 37 microns; Clavius could be fitted with the ash of sizes less than 37 microns, and the furnace slag less than 1 micron.

The polarimetric properties of the furnace slag, volcanic ash, and additional laboratory samples were investigated for wavelength dependence. It was found that the best color-polarimetric and best 
visual photometric match to Crisium were obtained with coarse chunks of the volcanic ash topped with less than 1 micron particles of itself.

This work is supported by NASA under Contract NAS 9-4942. 


\section{INTRODUCTION}

Polarimetric studies of rough surfaces serve to delimit their physical and geometric properties. A theoretical approach to polarimetry of rough surfaces is extremely difficult, and an experimental approach can be pursued more readily, a1though not without difficulties. Thus, in an effort to lay the basis for an analytical approach, the polarization properties of volcanic ash and furnace slag were investigated as a function of particle size, albedo and porosity; current theories of polarization indicate that these properties are significant. 


\section{DISCUSSION}

For the volcanic ash, coarse particles (of the order of millimeters in size) produced too high a maximum polarization to match the lunar maria or highlands. Smaller particles (Fig. 1) between 37 and 88 microns in size matched the Crisium curve of Gehrels et al., and particles less than 37 microns matched Clavius. Furnace slag with particles less than 37 microns matched Crisium and particle sizes less than 1 micron matched Clavius.

One possibility for a good photometric match is large particles of macroporous structure (for instance volcanic ash), to produce the necessary shadowing. Thus, a combination of the coarse material and a powder of the same material on the surface should produce the proper polarimetry and photometry. The polarimetry of a contrived model of Haleakala volcanic ash is shown in

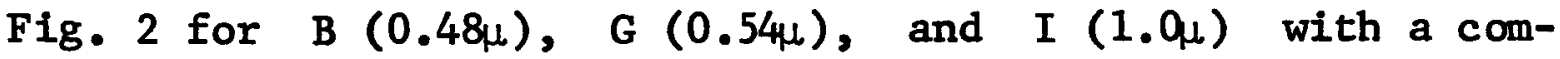

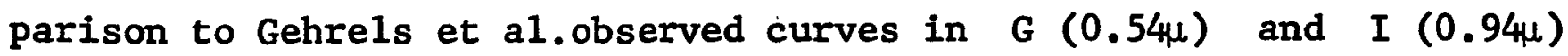
for Crisium. A fair fit is obtained for G, but the $I$ is low, possibly the result of a longer effective wavelength in our system.

The photometric function for the composite surface is good (Fig. 3). The shaded area is a compilation of lunar data from Fedoretz-Orlova and Minnaert-Van Diggelen. 
For six contrived polarimetric models of the lunar surface (results depicted in Fig. 4), it is shown that the closest match to Clavius for maximum polarization versus wavelength is achieved with the slag on coral (Sample No. 2), and the closest match to Crisium with the volcanic ash topped with less than 1 micron of itself (Sample No. 5); No. 6, another Haleakala volcanic ash topped with less than 1 micron of itself, also is a comparatively good fit. However, the composite coral (Sample No. 2) produced a poor match in the polarization-wavelength curves not presented here. Furnace slag particles on larger chunks (Sample No. 7) was a poor match as well as coral particles less than 1 micron sprinkled on a volcanic ash (Sample No. 4).

Work is continuing on contrived models as part of a program of definition of the lunar surface in terms of an interdisciplinary integrated approach, using not only photometric and polarimetric data, but thermal, radio-radar and mechanical data. 


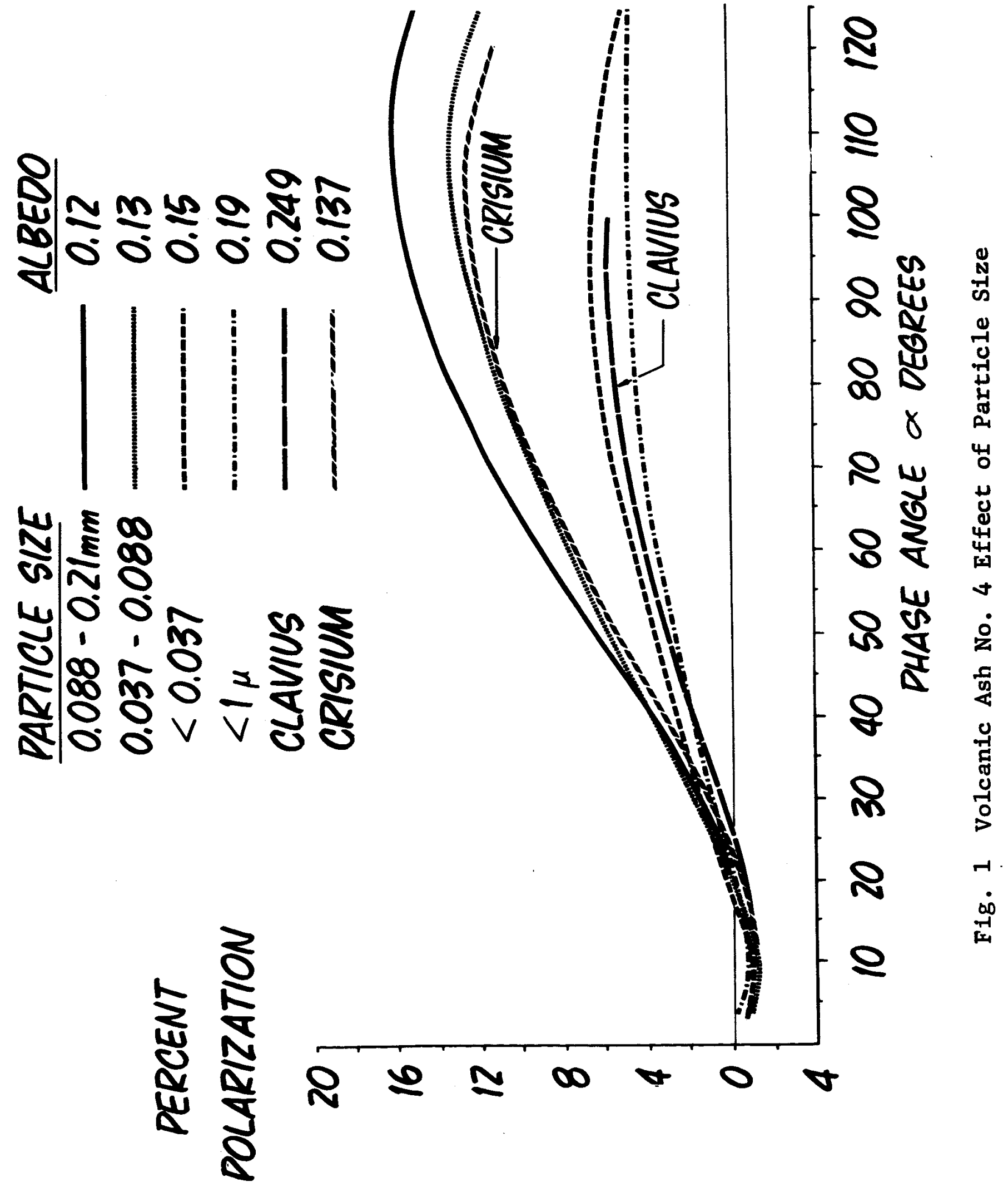




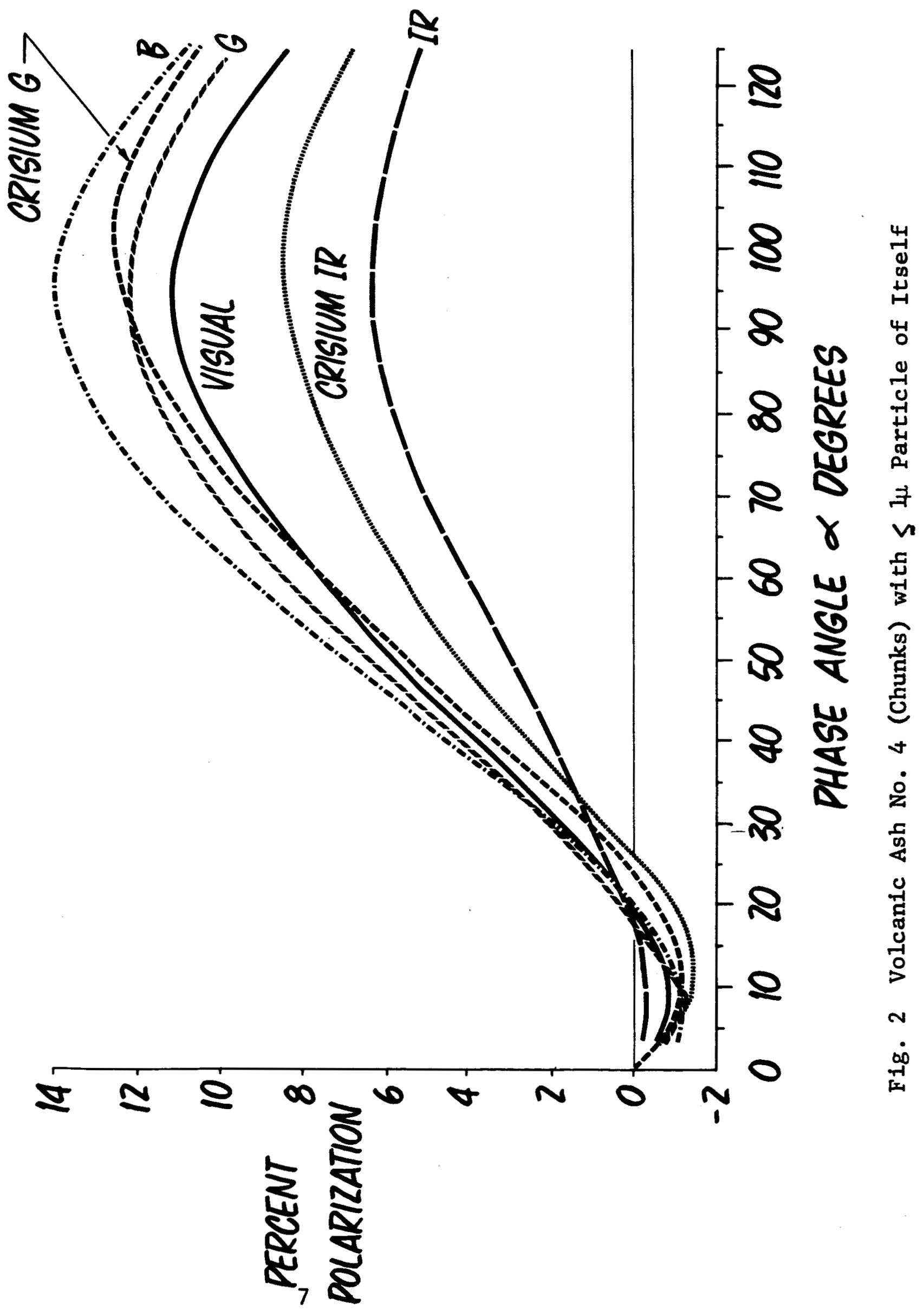




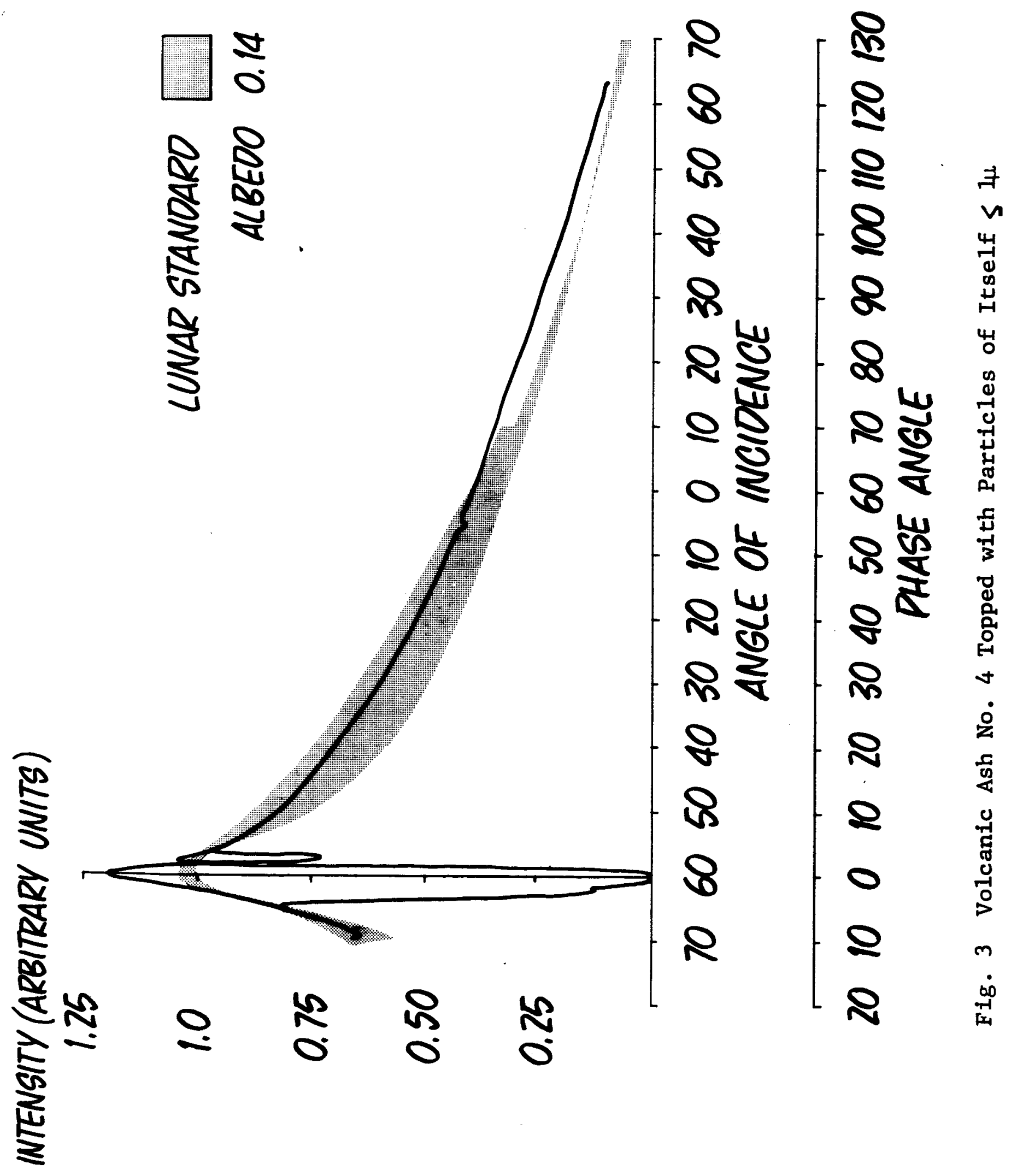




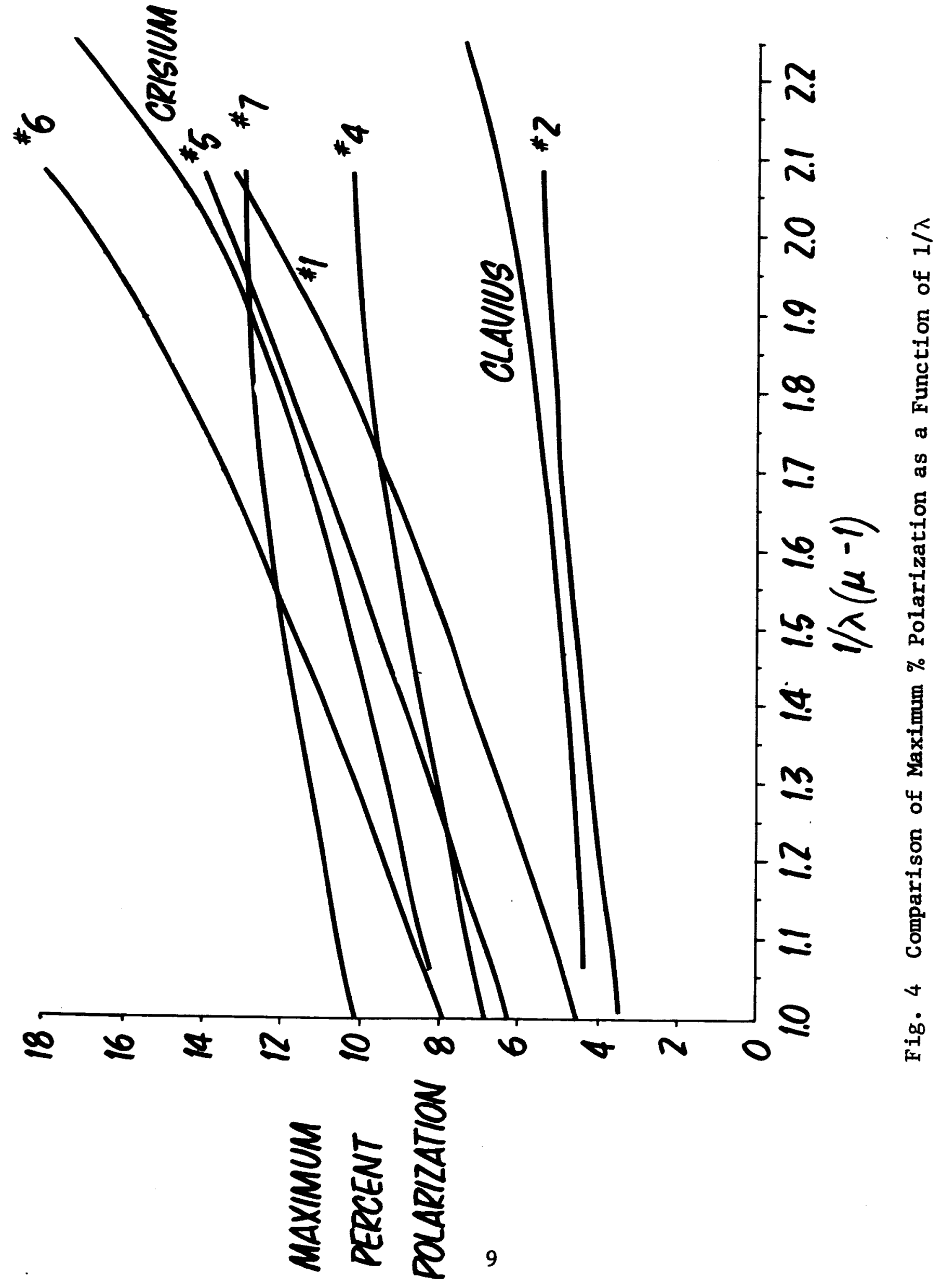




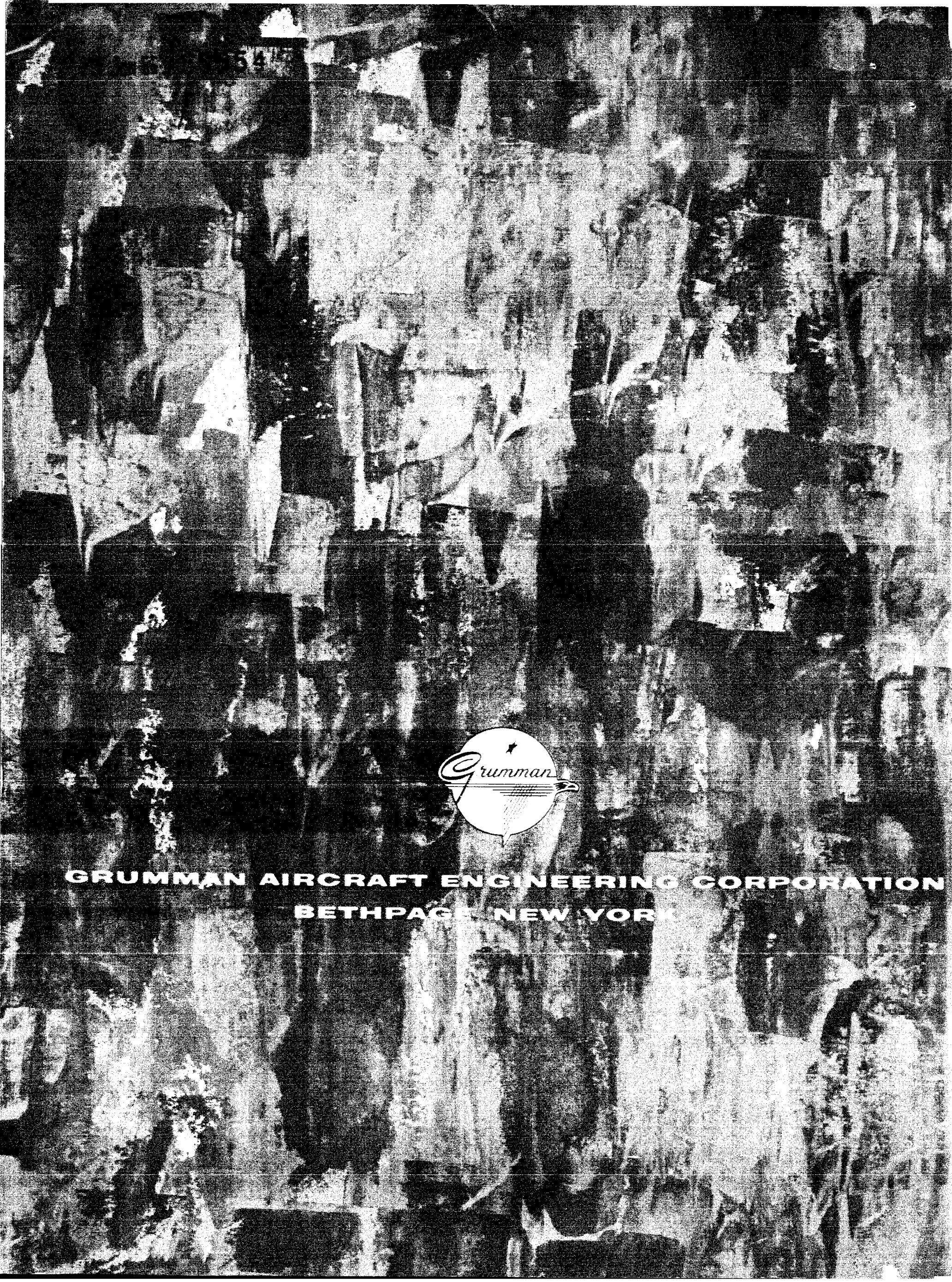

